\title{
Awareness about diabetes mellitus and DKA among medical students: an observational study
}

\author{
Manu G. ${ }^{1}$, Narasimhamurthy K. M.*2
}

${ }^{1}$ Department of Pharmacology, Adichunchanagiri Institute of Medical Sciences, B G Nagar, Nagamangala Taluk, Mandya 571448, Karnataka, India ${ }^{2}$ Department of Pharmacology, Basaveshwara Medical College and Hospital, Chitradurga 577502, Karnataka, India

Received: 16 October 2018 Accepted: 19 November 2018

\section{*Correspondence to:}

Dr. Narasimhamurthy K. M., Email: drmanugigu@gmail.com

Copyright: (C) the author(s), publisher and licensee Medip Academy. This is an openaccess article distributed under the terms of the Creative Commons Attribution NonCommercial License, which permits unrestricted noncommercial use, distribution, and reproduction in any medium, provided the original work is properly cited.

\begin{abstract}
Background: Diabetes Mellitus (DM) is a global epidemic and a leading cause for increased mortality and morbidity. The prevalence of Diabetes is very high so the management of DM and its complication like diabetic ketoacidosis (DKA) is very crucial in today's world. Medical students being the future pillars of our health care system were included in this study to know their knowledge towards diabetes and its acute complication DKA.

Methods: This study was a questionnaire based observational study conducted in Adichunchanagiri Institute of Medical Sciences, B G Nagara. Final year medical students and interns were included in the study.

Results: A total of 150 (75 from each group) questionnaires were collected and analysed. Most of the final year students and interns were aware about the classical symptoms of diabetes $(73.33 \%$ and $84 \%$ respectively) and were aware about the endocrine gland related to diabetes $(82.6 \%$ and $94.6 \%$ respectively) however there were differences about the meaning of PPBS among both the groups. Interns $(76 \%)$ had better knowledge regarding the fluid replacement in the management of DKA in compared to final year students $(41.33 \%)$. There was statistically significant difference in knowledge between the two groups regarding GDM.

Conclusions: This study identifies that both final year students and interns need to improve the practical knowledge towards diagnostic parameters of DM and treatment of DKA. Continous medical education programmes and workshops should be organised to enhance the knowledge towards DM and its complications.
\end{abstract}

Keywords: Awareness about diabetes mellitus, DKA, Medical students

\section{INTRODUCTION}

Diabetes Mellitus (DM) is a global epidemic and a leading cause for increased mortality and morbidity. ${ }^{1}$ According to International Diabetes Federation estimates, in 2015 around 415 million people had DM and this number is expected to rise by 2040 to 642 million. India is home to 69.1 million people with DM and is estimated to have the $2^{\text {nd }}$ highest number of cases in the world of DM after China in 2015. ${ }^{2}$ Rapid cultural and social changes including ageing population, increasing urbanization, dietary changes, unhealthy behaviour and reduced physical activity are the causes to increase in DM. The incidence and prevalence of DM is increasing in India's urban middle class and working poor accordingly to recent epidemiological evidence. ${ }^{3}$ Indians also have a greater degree of insulin resistance and a stronger genetic predisposition to DM. ${ }^{4}$ So, WHO has declared India as the "Diabetic Capital" in view of the above facts. ${ }^{5}$

The factors explaining the failure of early diagnosis are inadequate knowledge, public awareness about symptoms of DM, its complications and prevention, lack of 
infrastructure for screening DM and lack of identification of high risk group resulting in development of DM in young individuals suffering from chronic morbidities ending up living a poor quality of life. ${ }^{6}$

Health professionals play a vital role in improving the quality of healthcare for people with DM. The knowledge, education and awareness of the diabetes among medical undergraduates are essential because in future they are going to tackle this major public health problem. ${ }^{7}$ Effective management of DM will reduce the incidence and progression of DM and its complications. Medical student's being the future general practitioners or family physicians are the one to who people with symptoms of diabetes will approach to. Hence, it is important to impart scientific knowledge about diabetes among medical students and incorporate it in medical education curriculum in most effective way. This study was aimed to evaluate the awareness of medical students regarding diabetes mellitus.

\section{METHODS}

The design of the study was a questionnaire based observational study. A study carried out at Adichunchanagiri Institute of Medical Sciences (AIMS), B G Nagara. The study was conducted during January 2017 to February 2017.

\section{Inclusion criteria}

Final year medical students and Interns who were willing to participate were included in the study.

\section{Exclusion criteria}

Medical students and interns who were not willing to participate were excluded from the study.

The purpose of the study was explained to the respondents and informed consent was taken. The participants were assured that the outcome would not be revealed or be used for performance appraisal of the students. A feedback questionnaire covering various aspects of Diabetes and its complication DKA was distributed among the participants.

The questions were regarding general information of diabetes (classical symptoms of diabetes, which endocrine gland is related to diabetes, which would be the world's capital for diabetes by 2035 , when is the world's diabetes day celebrated), best indicator of glycaemic control (is it glycosylated haemoglobin or Fasting blood sugar) and regarding DKA (is DKA an acute complication of diabetes, which electrolyte is commonly depleted in DKA, is short acting insulin used in DKA) and regarding gestational diabetes (which is the initial test done to evaluate GDM, which is the mode of treatment). The filled questionnaire feedbacks were retrieved from the participants.

\section{Statistical analysis}

The returned questionnaires were checked for completeness of the data and the descriptive data were expressed in percentages.

Quality control was maintained as per the standard protocol. Confidentiality was maintained during the study.

\section{RESULTS}

A total of 150 (75 from each group) questionnaires were collected and analysed. Most of the final year students and interns were aware about the classical symptoms of diabetes $(73.33 \%$ and $84 \%$ respectively) and were aware about the endocrine gland related to diabetes $(82.6 \%$ and $94.6 \%$ respectively). $48 \%$ of final year students and $62.6 \%$ interns told that India would be the world capital for diabetes but rest all mentioned China would be (Figure 1).

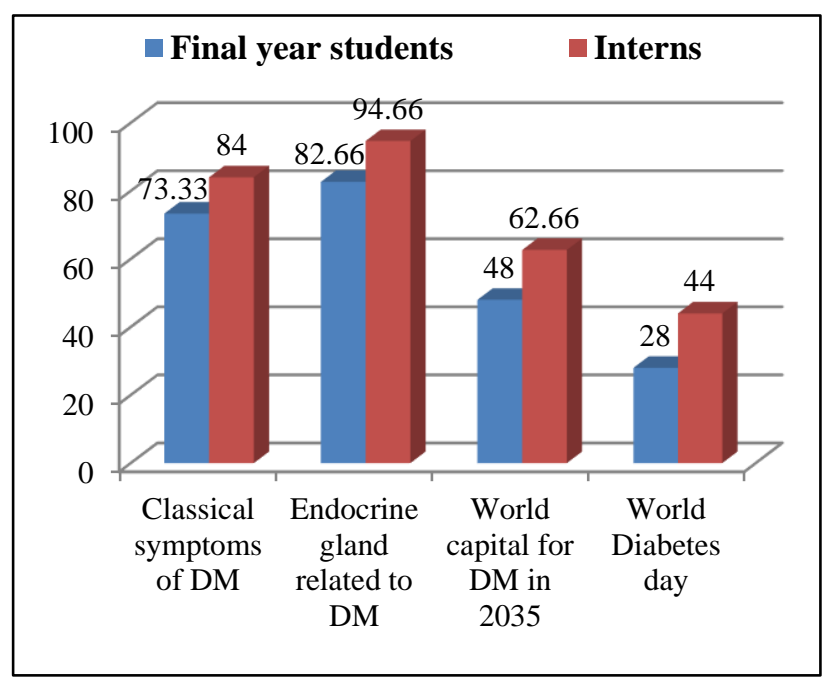

Figure 1: Questions on general information of DM.

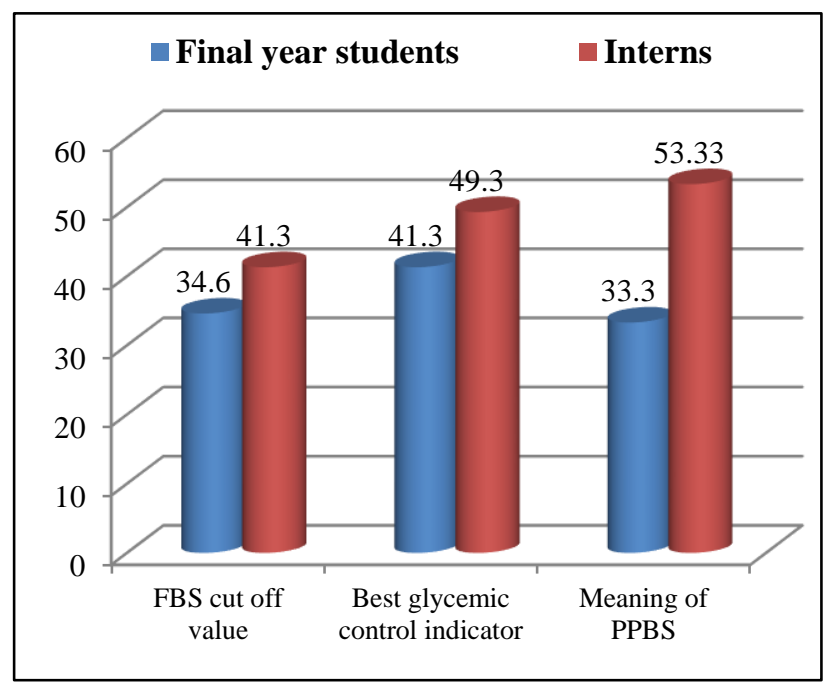

Figure 2: Questions on biochemical parameters of DM. 
The awareness on FBS cut off value and the best glycemic control indicator for diabetes among both the groups were not that much different however there were differences about the meaning of PPBS among both the groups (Figure 2).

There was not much difference in the knowledge regarding DKA among final year students and interns, however interns $(76 \%)$ had better knowledge regarding the fluid replacement in the management of DKA (Figure 3) in compared to final year students $(41.33 \%)$.

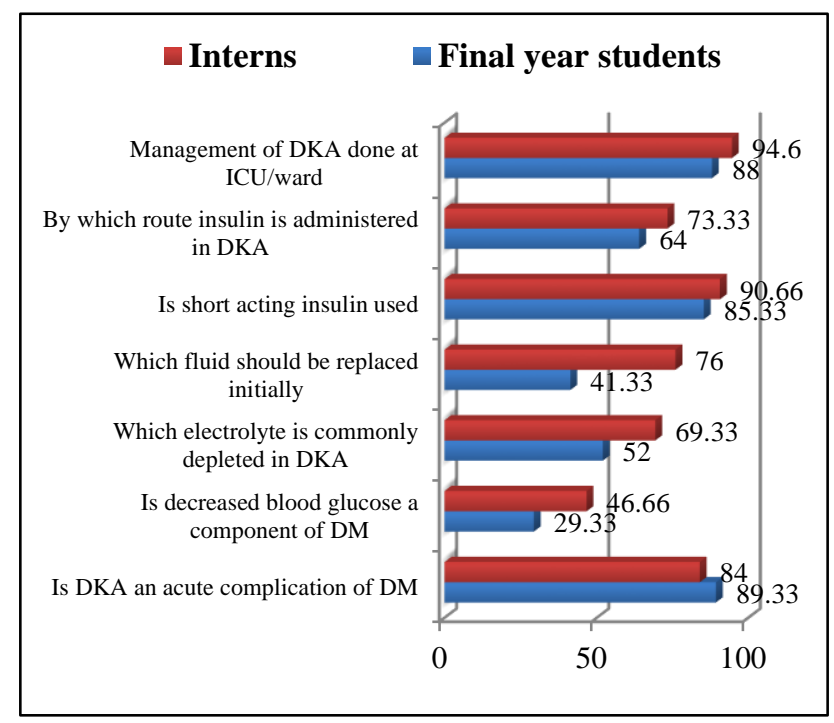

Figure 3: Questions on DKA.

There was statistically significant difference in knowledge between the two groups regarding GDM (Figure 4). Interns had more knowledge regarding the initial evaluation test done in GDM and also regarding the mode of treatment in GDM in compared to final year students.

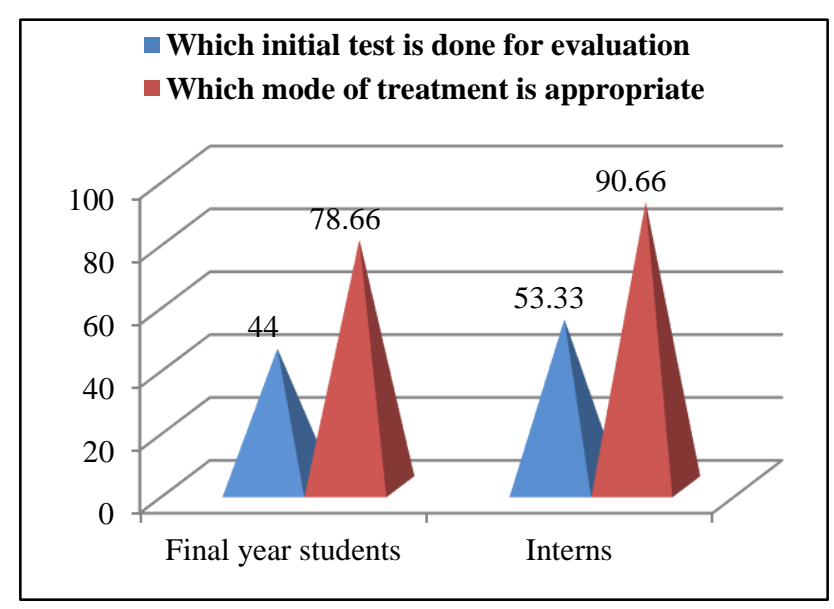

Figure 4: Questions on GDM.

Figure 5 shows the overall correct response given by both final year students and interns where it shows that both the groups are knowledgeable regarding general information of diabetes mellitus; however, there is difference in their knowledge regarding the biochemical parameters, management of DKA and GDM.

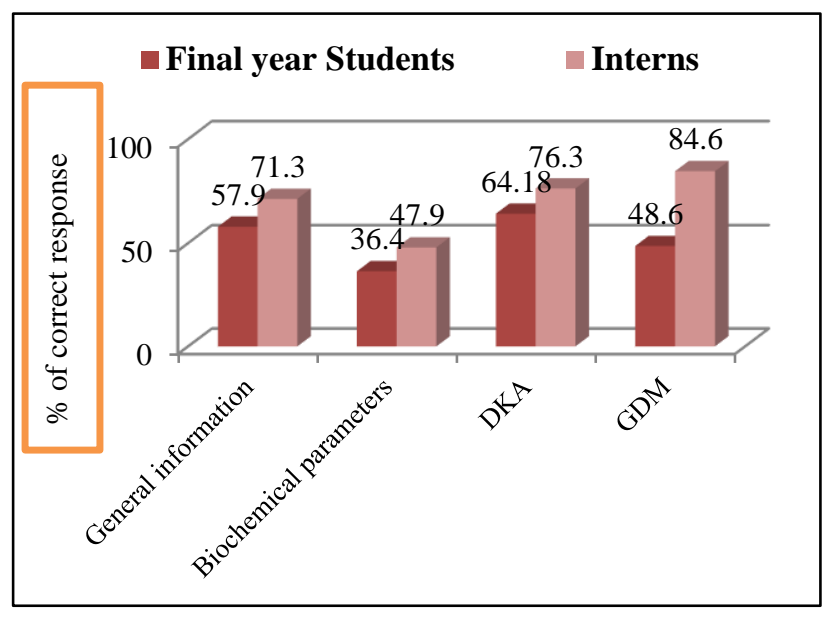

Figure 5: Correct responses among both the groups.

\section{DISCUSSION}

Our study aimed to study the awareness of diabetes mellitus among medical students. Not many studies have been done so far regarding awareness of diabetes among undergraduate medical students. Most of the final year students and interns were able to tell the classical symptoms of DM and the endocrine gland related to DM as pancreas. However not many anticipated that India would be the world capital of Diabetes by 2035 and were not aware about the World Diabetes day. Many students said the cut of value for FBS as $110 \mathrm{mg} / \mathrm{dl}$ and mentioned FBS to be the best indicator of glycaemic control instead HbA1c. DKA the acute complication of DM-1 was considered to be of Type 2 DM by many students. The initial test for evaluation of GDM patient most of them mentioned as glucose tolerance test instead glucose challenge test. A similar study conducted in Mangalore among medical students, it was found that $60 \%$ of final year students and $71.5 \%$ interns had good knowledge about DKA. ${ }^{8}$ In a study conducted in Tamil Nadu, only $50 \%$ of the final year medical students were aware about DKA. ${ }^{9}$ A study conducted in King Faisal Medical College in Saudi states that students had poor awareness about epidemiology of diabetes and diabetes practices. ${ }^{10}$ In this study, the awareness and knowledge about DM was better in Interns when compared to final year students.

\section{CONCLUSION}

This study identifies that both final year students and interns had basic knowledge about diabetes mellitus however they need to improve the practical knowledge of medical students towards diagnostic parameters and treatment of DKA. Continous medical education programmes and workshops should be organised to enhance the knowledge towards DM because they are the future health care providers to screen and manage diabetes in the community. 
Funding: No funding sources

Conflict of interest: None declared

Ethical approval: The study was approved by the

Institutional Ethics Committee

\section{REFERENCES}

1. Wild S, Roglic G, Green A, Sicree R, King H. Global prevalence of diabetes: estimates for the year 2000 and projections for 2030. Diabetes Care. 2004;27:1047-53.

2. International Diabetes Federation. In: Cavan D, Fernandes JR, Makaroff L, Ogurtsova K, Webber S, editors. Diabetes Atlas. $7^{\text {th }}$ ed. Brussels, Belgium: International Diabetes Federation; 2015:51-65.

3. Mendenhall E, Shivashankar R, Tandon N, Ali MK, Narayan KMV, Prabhakaran D. Stress and diabetes in socioeconomic context: a qualitative study of urban Indians. Soc Sci Med. 2012;75(12):2522-9.

4. Mohan V. Why are Indians more prone to diabetes? J Assoc Physicians India. 2004;52:468-74.

5. Kokiwar PR, Gupta S, Durge PM. Prevalence of diabetes in a rural area of Central India; Int. J. Diab Dev Ctries. March 2007;27(1):8-10.

6. Raheja BS, Kapur A, Bhoraskar A, Sathe SR, Jorgensen LN, Moorthi SR, et al. Diab Care Asia-India Study: Diabetes care in India-Current status. J Assoc Physicians India. 2001;49:717-22.
7. Trepp R, Wille T, Wieland T, Reinhart WH. Diabetesrelated knowledge among medical and nursing house staff. Swiss Med Wkly. 2010;140(25-26):370-5.

8. Nazareth RA, Arunachalam R, Sudeep K. Study of awareness about diabetes mellitus among undergraduate medical students. Int J Rec Tren Scie Technol. 2014 Oct;12(3):491-3.

9. Singh H, Thangaraju P, Kumar S, Aravindan U, Balasubramanian H, Selvan T. Awareness of Diabetes and Diabetic Ketoacidosis (DKA) Among Medical Students in a Ter Hospital: An Observational Study. J Clin Diagn Res. 2014;8:4-6.

10. Wadaani FA. The knowledge attitude and practice regarding diabetes and diabetic retinopathy among the final year medical students of King Faisal University Medical College of Al Hasa region of Saudi Arabia: a cross sectional survey. Nig J Cli Practi. 2013;16(2):164-8.

Cite this article as: Manu G, Narasimhamurthy KM. Awareness about diabetes mellitus and DKA among medical students: an observational study. Int J Basic Clin Pharmacol 2019;8:111-4. 